\title{
Flat Directions in the Early Universe: Birth, Life and Death
}

\section{Francesco RIVA*}

Rudolf Peierls Centre for Theoretical Physics, University of Oxford, Oxford, UK and Theory Division, Physics Department, CERN, Geneva, Switzerland

E-mail: riva@thphys.ox.ac.uk

\begin{abstract}
We shortly review the implications of supersymmetric flat directions for post-inflationary cosmology. Beside providing a mechanism for baryogenesis à la Affleck and Dine, the large vacuum expectation values (VEVs) of fields along MSSM flat directions could play an important role during thermalization. If they decay after the inflaton, they could in principle delay reheating or even come to dominate the energy density of the entire universe. The cosmological fate of flat directions is therefore of primary importance in determining the value of the reheat temperature, which sets the initial conditions for standard Hot Big Bang cosmology. We review the evolution of these condensates during the early universe, governed by an effective potential arising from Supersymmetry breaking and non-renormalizable terms. A consequence of the resulting motion of flat direction's VEVs is its non-perturbative decay, in a mechanism similar to inflationary preheating. The time scale of this decay is much shorter than the timescale for decay of a gravitationally coupled inflaton and, consequently, flat directions will not delay thermalization.
\end{abstract}

Cargèse Summer School: Cosmology and Particle Physics Beyond the Standard Models July 30 - August 11, 2007

Institut d'Etudes Scientifiques de Cargèse

*I am very thankful to the organizers of the Cargèse Summer School and to Stephen West for useful comments on the first draft of this talk. This research project has been supported by the Greendale Scholarship at Merton College, Oxford, and by the Marie Curie Early Stage Training Fellowship of the European Community's Sixth Framework Programme under contract number (MEST-CT-2005-020238-EUROTHEPHY). 


\section{Implications of Flat Directions for the Early Universe}

Supersymmetry (SUSY) and gauge symmetries allow only certain types of interaction to appear in the scalar potential. In the limit of unbroken SUSY and vanishing non-renormalizable terms, the potential for a scalar field $\Phi_{i}$ charged under gauge symmetries with generators $T^{a}$ and coupling constants $g_{a}$ can be written as

$$
V=\sum_{i}\left|F_{i}\right|^{2}+\frac{1}{2} \sum_{a} g_{a}^{2} D_{a} D_{a}, \quad \text { with } \quad F_{i}^{*}=\frac{\partial W}{\partial \Phi_{i}} \quad \text { and } \quad D_{a}=\sum_{i} \Phi_{i}^{\dagger} T^{a} \Phi_{i},
$$

where $W$ is the superpotential, an analytic function of the $\Phi_{i}$ 's. Differently form the Standard Model, along special directions in field space the constrained SUSY potential from eq.1.1 exactly vanishes, and large vacuum expectation values (VEVs) can develop with no cost in energy, these are called flat directions (FDs). In the MSSM, one example is $L L e^{c}$, with complex scalar field VEVs $\phi$ given as $L_{e}=(0, \phi), L_{\mu}=(\phi, 0)$ and $e_{\tau}^{c}=\phi$. In this case the different families of the fields imply that $F=0$, while the fact that the combination $L L e^{c}$ is a gauge singlet gives $D=0$.

The presence of such FDs has a number of consequences for the history of the early universe [1]. As sugested by Affleck and Dine, some of these FD VEVs carry baryon number and their out of equilibrium CP violating decay provides a possible mechanism for baryogenesis [2]. Another important implication of FDs is that, if their VEVs are enough long-lived to decay after the inflaton, then thermalization of the universe (reheating) can be delayed. This is because fields coupled to the FD acquire large masses when the FD gets a VEV, thus blocking the decay of the inflaton into formerly light fields (both perturbatively and non-perturbatively [3]). Also, in models with a vanishing superpotential, $W=0$, (or models [3] in which inflation takes place along a FD) the energy stored in the FDs can come to dominate the energy density of the universe and therefore reheating has to wait for these FDs to decay [4]. The temperature at which the universe thermalizes sets the initial conditions for Standard Hot Big Bang cosmology and needs to be in agreement with the predictions of Big Bang Nucleonsynthesis, hence the importance of studying decay of FD VEVs.

The above arguments hold so long as the FD VEVs decay slowly enough. This is generally the case because fields coupled to the FD acquire large masses when the FD gets a VEV, consequently perturbative decay of the VEV into light fields can take place only via loops involving heavy fields. We can estimate [4] the decay rate of the FD $\Gamma_{\phi} \sim g^{4} \frac{m_{\phi}^{3}}{\langle\phi\rangle^{2}}$, where $g$ is the coupling of the flatdirection to some fields with mass given by the FD's VEV, $m \sim\langle\phi\rangle ; m_{\phi} \sim 1 \mathrm{TeV}$ is the mass of the FD arising from soft SUSY breaking (see next section). This can be compared with the perturbative decay rate for a gravitationally coupled inflaton $\Gamma_{\psi} \sim \frac{m_{\psi}^{3}}{M^{2}}$, where $M \sim 10^{19} \mathrm{GeV}$ is the Plank mass and $m_{\psi} \sim 10^{13} \mathrm{GeV}$ is the inflaton mass. In this case, a VEV of order $\mathscr{O}(100) \mathrm{TeV}$ is sufficient to delay the FD's decay untill after the decay of the inflaton, hence postponing thermalization.

\section{Cosmological Evolution of Flat Directions}

However, the world is not supersymmetric and the scale of gravity is finite: flat directions are not exactly flat! The effective scalar potential along a generic FD $\phi$ is given by [5]

$$
V \supset\left(m_{\text {susy }}^{2}-c H^{2}\right)|\phi|^{2}+\lambda \frac{A m_{\text {susy }}+a H}{d M^{d-3}} \phi^{d}+\frac{|\lambda|^{2}}{M^{2 d-6}}|\phi|^{2 d-2},
$$


where $m_{S U S Y}$ is the soft SUSY breaking mass along the FD and the term $c H^{2}$ is a result of the SUSY breaking due to the finite energy density of the early universe (here we consider the case $c \sim \mathscr{O}(1)>0$, where the minimum of the potential is displaced from the origin); $H$ is the Hubble constant. The second term also includes soft (or due to the finite energy density) SUSY breaking A-terms, $d$ is the dimension of the lowest order terms appearing in the scalar potential, which depends on the particular $R_{p}$-parity and gauge symmetry assignments for the fields in the FD; $\lambda$, $A$ and $a$ are dimensionless coefficients. The last term comes from non-renormalizable terms in the superpotential, its role is to bound the minimum of the scalar potential to the region $\langle|\phi|\rangle \ll M$.

Note that the second term depends explicitly on the complex phase of the FD; this term is responsible for the violation of baryon number (if the FD carries baryon number) necessary for baryogenesis. As we will see shortly, this term also leads to the particular dynamics of the FD's VEV that will eventually cause its non-perturbative decay.

Let's now concentrate on the dynamics of the FD's VEV, governed by the scalar potential 2.1 and the equation of motion for a scalar field in a deSitter background

$$
\ddot{\phi}+3 H \dot{\phi}+V^{\prime}(\phi)=0,
$$

where dots represent time derivatives and primes field derivatives. During inflation, the finite energy term dominates, $V^{\prime \prime} \approx H^{2}$, and the field is parametrically near critically damped: it quickly evolves to the minimum of the potential [5]

$$
\left|\phi_{\min }\right|=\left(\frac{\beta H M^{d-3}}{\lambda}\right)^{\frac{1}{d-2}}
$$

where $\beta$ is a numerical constant depending on $a, c$ and $d$. Here $H$ is the Hubble constant during inflation and for generic FDs, $\left|\phi_{\text {min }}\right| \sim \mathscr{O}\left(10^{16}\right) \mathrm{GeV}$. The phase $\sigma=\operatorname{Arg}(\phi)$ of this minimum, on the other hand, is set by the A-term: its potential is of the form $V(\sigma) \propto \cos \left(\theta_{a}+\theta_{\lambda}+d \sigma\right)$, where $\theta_{a}$ and $\theta_{\lambda}$ are the complex phases of the terms appearing in eq.2.1. It is important to note that the initial (post-inflationary) value of the FD's VEV is completely determined by the scalar potential eq.2.1 and not by random fluctuations.

After inflation, $H$ decreases and the minimum of the potential also decreases as in eq.2.3. When $H \sim m_{\text {susy }}$, the soft SUSY breaking terms become important: the minimum of the potential is now displaced to the origin and, since the A-term and mass term are of a comparable size, the condensate develops angular motion. Finally, when $H<m_{\text {susy }}$ the potential reduces to $V \approx$ $m_{\text {susy }}^{2}|\phi|^{2}$. The FD's VEV rotates in the complex plane with constant angular velocity while its amplitude still decreases due to the Hubble expansion. Eventually the FD will decay perturbatively as explained in the previous section.

\section{Non-Perturbative Flat Direction Decay}

As originally described in $[6,7]$ there is however a much quicker way for a condensate to decay: non-perturbatively via a mechanism known as preheating. The basic idea is that a real scalar VEV $\psi$ (for example, the inflaton) undergoing classical oscillations $\psi(t) \sim \psi_{0} \sin (\omega t)$ with frequency $\omega$ and coupled to some quantum excitation $\chi$ via the Lagrangian term $g^{2} \psi^{2} \chi^{2}$ gives a time-dependent contribution $g^{2} \psi(t)^{2}$ to the effective mass of $\chi$. When considering the equations of motion for the field $\chi$ (which eventually leads to a definition of particles via expansion of the quantum field in terms of creation and annihilation operators), one identifies instabilities depending 
on the value of the time-derivative $\dot{\psi}$ when $\psi(t)$ passes through the origin. These instabilities correspond to a resonant, non-perturbative production of quanta of the field $\chi$ which, in turn, signal the fast decay of the scalar VEV responsible for this production.

In the framework of FDs, however, preheating as described above is generally inefficient. The main reason is that, differently from the inflaton, the flat-direction $\phi$ is a complex field and, as discussed in the previous section, undergoes a circular motion in the complex plane. Couplings of the form $|\phi|^{2}|\chi|^{2}$ coming from $F$-terms in the scalar potential give rise to effective mass terms $\sim|\phi|^{2}$ for the quantum field $\chi$. Now, due to its circular (eliptic) motion, the time derivative of the FD amplitude $d|\phi| / d t$ is in general very small (unless the orbit is squeezed along a particular line in the complex plane) and effective resonance production of particles takes place only with fine-tuned conditions [8].

There is however another effect [9] that plays an important role in FD preheating, due to the angular motion of the flat-direction VEV [4] and the presence of several light excitations around it. The scalar potential coming from $D$-terms includes a mass-matrix term for these excitations around the VEV, which has time-dependent eignevectors, including the heavy Higgs field and the massless Golstone boson of the broken gauge symmetry, together with other light excitations around the FD's VEV. In the unitary gauge [10], it is this time-dependence (mixing the Higgs field with the physical light excitations) that is now responsible for the non-perturbative production of particles, similarly to time-dependent mass in single-field preheating.

It can be shown [10] that a necessary condition for FDs to preheat is that more than one physical (i.e. not removable via a symmetry transformation) complex phase of the FD's VEV has a non-trivial dynamics after inflation. This is generally the case, provided there are enough fields making up the FD. Furthermore, since SUSY breaking and loop effects contribute phasedependent terms in the scalar potential, these phases tend to assume non-trivial values during the early universe. Although the details of the decay depend on the particular FD and on the SUSY breaking mechanism chosen, these non-perturbative effects will lead to the fast depletion of the VEV once the FD starts rotating. In models with a gravitationally coupled inflaton field the FD starts its circular motion long before inflaton decay. This brings us to conclude that, although FDs play an important role in baryogenesis, they won't delay the thermalization of the universe.

\section{References}

[1] T. Gherghetta, C. F. Kolda and S. P. Martin, Nucl. Phys. B 468, 37 (1996). For a review, see K. Enqvist and A. Mazumdar, Phys. Rept. 380, 99 (2003).

[2] I. Affleck and M. Dine, Nucl. Phys. B 249, 361 (1985), A. D. Linde, Phys. Lett. B 160, 243 (1985).

[3] See eg., R. Allahverdi and A. Mazumdar, [arXiv:hep-ph/0603244], R. Allahverdi, K. Enqvist, J. Garcia-Bellido and A. Mazumdar, Phys. Rev. Lett. 97 (2006) 191304.

[4] K. A. Olive and M. Peloso, Phys. Rev. D 74, 103514 (2006). See also references therein.

[5] M. Dine, L. Randall and S. D. Thomas, Phys. Rev. Lett. 75, 398 (1995).

[6] L. Kofman, A. D. Linde and A. A. Starobinsky, Phys. Rev. D 56 (1997) 3258.

[7] Y. B. Zeldovich and A. A. Starobinsky, Sov. Phys. JETP 34 (1972) 1159.

[8] Z. Chacko, H. Murayama and M. Perelstein, Phys. Rev. D 68 (2003) 063515 [arXiv:hep-ph/0211369].

[9] H. P. Nilles, M. Peloso and L. Sorbo, JHEP 0104 (2001) 004.

[10] A. Basboll, D. Maybury, F. Riva and S. M. West, arXiv:hep-ph/0703015. 